\title{
Politiques d'accès aux soins et données épidémiologiques
}

\section{Access to Care Policies and Epidemiological Data}

\section{L.-M. Joly · C. Damm}

Reçu le 18 décembre 2021; accepté le 21 décembre 2021

(C) SFMU et Lavoisier SAS 2021

Comment inciter les patients à utiliser des solutions mieux adaptées à leur demande de soins et éviter ainsi qu'ils ne se rendent aux urgences ? C'est la question que beaucoup se posent depuis longtemps : les médecins urgentistes bien sûr, mais aussi tous les autres médecins, les décideurs politiques, les journalistes, les administrateurs et économistes de la santé ... et même peut-être une partie de nos concitoyens. À cette question, il n'existe sans doute pas de réponse unique. L'une des raisons qui peuvent expliquer les difficultés, voire les points de vue divergents, est que nous manquons cruellement de littérature scientifique médicale de qualité sur le sujet. Or, dans un régime démocratique, les décideurs en matière de santé doivent pouvoir disposer de données épidémiologiques fiables. En particulier, pour ce qui nous concerne, de travaux réalisés dans le contexte français, puisqu'il est difficile d'extrapoler au problème français une étude réalisée dans un autre pays dont le système de soins et les comportements anthropologiques sont différents.

Pour illustrer la difficulté du problème, rappelons le travail de Durand et al. [1], montrant qu'il n'existe aucune définition consensuelle dans la littérature médicale de ce qu'est un « patient non urgent », avec des variations de prévalence allant de 5 à $90 \%$ selon les études dans différents services d'urgences (variations qui peuvent correspondre à des populations différentes, mais aussi et surtout à des définitions très variables d'une étude à l'autre, avec souvent une caractérisation a posteriori de l'urgence, puisque cet article montrait que les études étaient rétrospectives dans les deux tiers des cas). Les études qui apportent un éclairage sur ce problème de l'accès aux soins sont donc très attendues, surtout quand

\section{L.-M. Joly $(\bowtie)$}

Service des urgences adultes Charles-Nicolle,

CHU de Rouen, université de Rouen,

1, rue de Germont, F-76000 Rouen, France

e-mail : luc-marie.joly@chu-rouen.fr

C. Damm

Samu 76, CHU de Rouen,

1, rue de Germont, F-76000 Rouen, France elles sont françaises et quand elles présentent des données multicentriques issues de très grandes bases de données. C'est bien le cas de l'article de Mourou et al. [2] qui présentent dans ce numéro des Annales françaises de médecine d'urgence des données issues des résumés de passage aux urgences (RPU) sur toute la région Occitanie, couvrant trois années pleines de 2016 à 2018, et totalisant plus de 1,8 million de passages. Cette étude épidémiologique transversale décrit les caractéristiques de deux groupes de patients : les $80 \%$ qui se présentent spontanément aux urgences de leur propre initiative et les $20 \%$ qui y sont adressés après conseil médical (15\% par le Samu et $5 \%$ par un médecin libéral). Ces deux groupes apparaissent fort différents : les patients venant spontanément ont en moyenne 20 ans de moins que ceux adressés par un médecin ; leur temps de passage aux urgences est presque deux fois plus court, et ils présentent rarement une pathologie grave, avec $0,4 \%$ seulement de CCMU 4 ou 5 (contre $3,2 \%$ pour les patients adressés). Ils sont, enfin, trois fois moins souvent hospitalisés que les patients adressés par un médecin. Ces résultats sont à mettre en perspective avec le fait que les motifs de consultation sont assez similaires dans les deux groupes, à l'exception des motifs traumatologiques, plus fréquents dans le groupe venant spontanément. La comparaison, au sein des patients adressés, entre le groupe adressé via la régulation SAMU et celui adressé par un médecin libéral, retrouvait par contre des groupes très similaires, peut-être, comme le discutent les auteurs, du fait d'un biais de classement entre ces deux groupes.

Cette étude est un bel exemple de travail sur des données administratives massives, mais elle en partage les limites habituelles : seuls les deux tiers des RPU de la région étaient assez complets pour être exploités, et surtout il n'a pas été possible de juger de la pertinence des passages aux urgences, ni dans un groupe ni dans l'autre. Même si l'on peut faire l'hypothèse raisonnable que les patients référés par un médecin ont de bonnes chances d'avoir un passage motivé aux urgences, il n'est pas possible de considérer a contrario que tous les patients venus spontanément auraient pu ou auraient dû éviter un passage aux urgences. Or, juger de la pertinence 
ou non d'un passage aux urgences est un exercice difficile. Il est relativement facile de juger a posteriori de la pertinence d'un passage au urgence, alors qu'on dispose du diagnostic, ou au moins au vu de la démarche qui a permis d'écarter les diagnostics graves. L'exercice est beaucoup plus délicat à l'arrivée aux urgences sur la simple « bonne ou mauvaise mine » du patient. Le tri à l'arrivée, à partir du motif de venue et des constantes vitales, permet, certes, d'isoler un groupe de patients à très bas risque, mais en aucun cas, il n'est recommandé de renvoyer ces patients « tri 5 » sans les prendre en charge. Dans une large étude américaine sur près de 35000 patients, Raven et al. [3] avaient identifié les patients pour lesquels un diagnostic bénin de sortie des urgences laissait penser qu'ils auraient pu, sans risque, être pris en charge en médecine de ville. Cependant, leurs motifs de venue aux urgences, codés à l'accueil, étaient largement partagés avec les autres patients. Et ces motifs de venue a priori peu graves étaient dans l'ensemble associés à plus de $10 \%$ de score de tri imposant une prise en charge immédiate, à plus de $10 \%$ d'hospitalisation et à 3,4\% d'indications opératoires en urgence. À l'échelon individuel, chaque médecin urgentiste a dans son expérience le souvenir de patients pris en charge aux urgences malgré un motif de venue pouvant a priori mériter d'une redirection vers la médecine libérale, mais dont le diagnostic s'est finalement révélé plus grave que prévu.

Il apparait donc qu'une fois que le patient est présent aux urgences, il est sans doute trop tard ou difficile de le refouler, et que le faire contre la volonté du patient peut constituer un problème éthique et une violation du code de santé publique. La solution doit donc se trouver en amont, et le projet de généralisation du service d'accès aux soins (SAS) en 2022 s'inscrit dans cette problématique. Actuellement testé sur 22 sites pilotes répartis dans 13 régions, le SAS a pour objectif de répondre 24 heures/24 à la demande de soins urgents ou de soins non programmés de la population en offrant un nouveau service d'orientation et de guidage dans le système de santé. L'efficacité du SAS repose sur la coordination ville-hôpital et notamment sur les communautés professionnelles territoriales de santé (CPTS). Le SAS, en s'appuyant sur des outils modernes de télémédecine tels la visioconférence ou l'accès instantané au dossier médical partagé du patient, devrait participer à réduire le nombre de patients adressés par le Samu aux urgences. L'amélioration de la qualité de la couverture mobile et l'intégration des outils de visioconférence dans un grand nombre de SamuSAS (avec dans le meilleur des cas un enregistrement des données dans le dossier de régulation médicale) permettront de réaliser un avis distant de qualité, voire dans l'avenir une véritable téléconsultation. Confirmer à distance qu'une petite plaie ne nécessite visiblement pas de points de suture ou prescrire à distance un antibiotique pour une infection urinaire simple contribuerait à réduire le nombre de patients orientés par défaut vers un service d'urgence. À l'appui de ces solutions innovantes surgissent des idées pour faciliter leur mise en ouvre. Par exemple celle initiée par l'association SAUV Life (https://sauvlife.fr/) de déployer des unités mobiles de télémédecine dans plusieurs départements français (Manche, Landes, Paris...). Celles-ci embarquent un secouriste et un infirmier ou un étudiant en médecine capables d'initier une téléconsultation avec un médecin distant et de servir de relais pour l'exécution des examens diagnostiques (électrocardiogramme, otoscopie, échographie...). Dans un avenir assez proche, la mise en place d'infirmiers en pratiques avancées de premiers recours qui, coordonnés par un médecin ou une équipe médicale, réaliseraient des actes dont les contours resteraient à définir est une autre option, et leurs décrets de compétence sont déjà parus. Dans l'état actuel des habitudes des Français, l'impact de ces solutions sera cependant modeste : l'étude de Mourou et al. [2] montre que cela ne concerne que $15 \%$ des patients qui viennent aux urgences. Le cœur de cible, ce sont en réalité les $80 \%$ de patients qui se rendent de leur propre chef dans un service d'urgence. On peut espérer qu'en incitant cette population (reste à voir comment...) à contacter le SAS, certains pourraient bénéficier d'un conseil médical ou d'une (télé) consultation évitant le passage aux urgences. Ce dispositif permettrait alors de réduire ce flux de patients venant spontanément aux urgences et d'améliorer le service rendu en termes de soins, tant pour le patient lui-même que pour les autres patients qui nécessitent une prise en charge dans des urgences non saturées. Cela repose cependant sur un redimensionnement important des plateformes Samu-SAS qui subissent, comme les services d'urgences, une augmentation inexorable du nombre d'appels. Véritable porte d'entrée à l'hôpital des patients non programmés, le SAS a pour objectif d'apporter à la population la réponse et l'orientation la plus adaptée. Il serait aussi l'interlocuteur privilégié des médecins libéraux qui souhaiteraient adresser leur patient aux urgences. Le SAS n'a en effet de sens qu'avec une meilleure coordination de l'ensemble des acteurs en médecine de ville : médecins généralistes, médecins spécialistes, infirmiers libéraux. Cette coordination en réseau de soins passe par un renforcement des liens villehôpital, et de manière plus large, par la mise en place de structures de type plateforme territoriale d'appui. C'est à ces conditions qu'on peut espérer qu'un SAS généralisé associé à la plateforme digitale permettra de réintégrer les patients dans un parcours de soins coordonné. Encore faudra-t-il disposer pour cela des effecteurs disponibles, ce qui n'est pas certain dans l'état actuel de la médecine libérale et des perspectives démographiques, le Conseil national de l'Ordre des médecins prévoyant pour les quatre prochaines années une diminution annuelle de 850 médecins généralistes $(-4 \%)$. 
Liens d'intérêts : les auteurs déclarent ne pas avoir de liens d'intérêts.

\section{Références}

1. Durand AC, Gentile S, Devictor B, et al (2011) ED patients: how nonurgent are they? Systematic review of the emergency medicine literature. AmJ Emerg Med 29:333-45
2. Mourou H, Latournerie G, Delisle E, Charpentier S (2021) En quoi les patients adressés dans les services d'urgences après avis médical sont-ils différents des patients venant d'eux-mêmes ? Ann Fr Med Urgence 11:357-65

3. Raven MC, Lowe RA, Maselli J, Hsia RY (2013) Comparison of presenting complaint vs discharge diagnosis for identifying "nonemergency" emergency department visits. JAMA 309:1145-53 